\title{
Combination of dietary pre-gelatinized starch and isomaltooligosaccharides improved pellet characteristics, subsequent feeding efficiencies and physiological status in African catfish, Clarias gariepinus, juveniles
}

\begin{abstract}
An 8-week study was conducted on the use of native tapioca starch (TS) or pre-gelatinized tapioca starch (PGTS), with or without the inclusion of isomaltooligosaccharides (IMO) at $0.5 \%$ on the growth, feeding efficiencies, muscle and plasma biochemical composition, intestinal short chain fatty acids (SCFA), differential cell counts, phagocytic ability/capacity, and liver glycogen content in African catfish, Clarias gariepinus. Each treatment was triplicated with each replicate consisting of 10 fish (initial weight of $6.2 \pm 0.3 \mathrm{~g}$ ). The bulk density (BD), pellet durability index (PDI), water stability (WS), water absorption index (WAI), water solubility index (WSI), and protein solubility (PS) were measured in all experimental diets. The results showed no significant $(\mathrm{p}>0.05)$ growth differences among the treatments, but feed intake was significantly lowest $(\mathrm{p}<0.05)$ in the PGTS diet, followed by the PGTS + IMO, while the significantly $(\mathrm{p}<0.05)$ highest was in the TS treatment. Catfish fed the PG diets had significantly $(\mathrm{p}<0.05)$ higher crude lipid but significantly $(\mathrm{p}<0.05)$ lower crude protein and ash compared to those fed the TS diets. Both trypsin and chymotrypsin activities were significantly $(\mathrm{p}<0.05)$ higher in the PG diets. The TS + IMO diet significantly increased the white blood cells and phagocytic activity compared to the TS diet. Meanwhile, fish fed the PGTS diet had significantly ( $\mathrm{p}<0.05$ ) lower intestinal acetic and butyric acid than all others, but the inclusion of IMO in the PGTS diet mitigated a decrease of these. The PG starch diets had significantly $(\mathrm{p}<0.05)$ higher PDI, WS, WAI, and PS than the TS diets. The uncompromised growth may have been due to dietary PG starch and IMO enhancing available energy to the fish. The mitigating effect of IMO on increasing intestinal SCFA when using PG diets may have implications to disease management, but requires further investigation.
\end{abstract}

Keyword: Prebiotic; Clarias gariepinus; Pre-gelatinized starch; Short chain fatty acids; Trypsin; Chymotrypsin; Pellet quality 